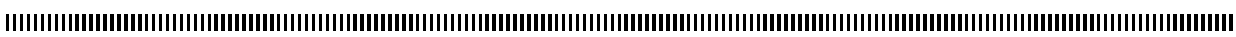

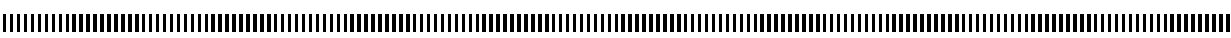
|

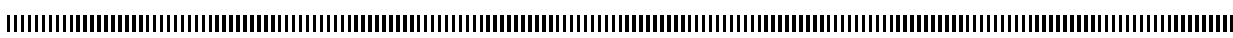

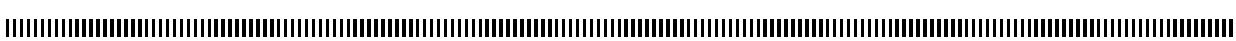
| |

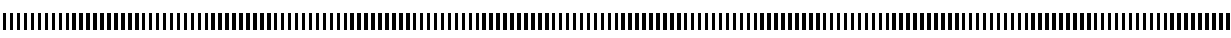
| |

\title{
Interval numerical observer: Application to a discrete time nonlinear fish model
}

\author{
Aboudramane Guiro *** — Abderrahman Iggidr ${ }^{* *}$ — Diéne Ngom ***** \\ * Laboratoire d'Analyse Mathématique des Equations (LAME) \\ UFR des Sciences exactes et Appliquées, Université de Ouagadougou \\ Ouagadougou, Burkina Faso \\ guiro@loria.fr \\ ** INRIA-Lorraine and LMAM-CNRS UMR 7122, University Paul Verlaine \\ Metz, France \\ iggidr@loria.fr, iggidr@math.univ-metz.fr \\ *** Laboratoire d'Analyse Numérique et d'Informatique (LANI) \\ UFR des Sciences Appliquées et de Technologie, Université Gaston Berger \\ Saint-Louis, Sénégal \\ ngomdiene@yahoo.fr, ngom@loria.fr, ngom@univ-metz.fr
}

|

ABSTRACT. The aim of this work is to reconstitute the state of a discrete-time nonlinear system representing a dynamical model of a harvested fish population. For this end, we are going to use a numerical method of building an interval observer for the consider discrete-time model fish population. We adapt to this model an algorithm called "Interval Moving Horizon State Estimation" (IMHSE) which gives an estimated interval of the system states. This algorithm is carried out in [8] and work well for a general class of discrete-time systems.

RÉSUMÉ. Le but de ce travail est de reconstruire les états d'un système discret non linéaire représentant la dynamique d'une population de poissons soumise à l'action de la pêche. Pour cela nous allons utiliser une méthode numérique de synthèse d'un observateur intervalle du modèle discret de la population de poissons considéré. Nous adaptons à ce modèle un algorithme appelé "Interval Moving Horizon State Estimation" (IMHSE) qui permet d'estimer les états du système par des intervalles. Cet algorithme est développé dans [8] et marche bien pour une classe générale de systèmes discrets.

KEYWORDS : Nonlinear discrete-time systems, interval observers, dynamic populations.

MOTS-CLÉS : Systèmes discrets non linéaires, observateurs intervalles, dynamique des populations.

1. Corresponding author: ngomdiene@yahoo.fr, ngom@loria.fr, ngom@univ-metz.fr

Volume 11 - Numéro spécial CARI 2008 - Editeur : Marc Kokou Assogba - Pages 37 à 52 - ARIMA

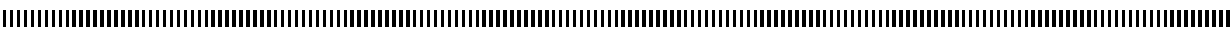

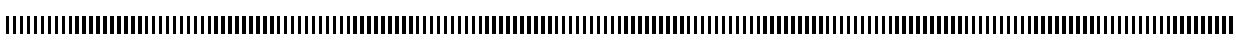
| |

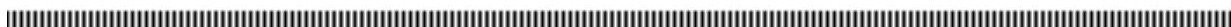




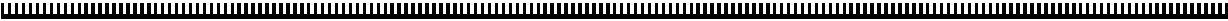

\section{Introduction}

Management of renewable resource has become nowadays one of the great priorities of political and economic decision-makers. In area of fishing, the difficulty in assessing stocks of available resource marine due to the complexity of oceans makes difficult the control of different species. Several mathematical models on dynamics evolution and exploitation of certain fish populations have been developed [6, 7, 16, 18, 21, 22]. These mathematical models combined with simulations on computer enable to observe, understand and regulate complex evolution of available resource marine. Basing oneself on existent mathematical models and using some tools of control theory, we may reconstruct the states of a dynamical system. Our aim in this paper is to evaluate the stock of an exploited fish population whose dynamics are represented by the following discrete-time nonlinear model (see for instance [7] page 76 ) :

$$
\left\{\begin{aligned}
x_{1}(k+1) & =f\left(\sum_{i=1}^{n} b_{i} x_{i}(k)\right) \\
x_{2}(k+1) & =x_{1}(k) \exp \left(-M_{1}-\tau q_{1} E(k)\right) \\
& \vdots \\
x_{n}(k+1) & =x_{n-1}(k) \exp \left(-M_{n-1}-\tau q_{n-1} E(k)\right)
\end{aligned}\right.
$$

Where

- $n$ is the number of age groups of the considered fish population.

- $k$ is the step time discretization.

- $x_{i}(k)$ is the number of individuals in age group $i(i=1,2, \ldots, n)$ at time $k$.

- $b_{i}$ is the fecundity rate of individuals in age group $i$.

- $M_{i}$ and $q_{i}$ are respectively death and capturability rate of individuals in age group $i$.

- $E(k)$ is the fishing effort at time $k$ and $\tau$ is the annual fishing time.

- $f$ is a recruitment function which is continuous and non decreasing.

The recruitment function is an approximation of the number of young fish that enter for the first time the exploitable phase. This recruitment function is very difficult to compute because of certain phenomena (temperature and depth of water, speed of wind,competition, predation, ... ) which influence growth of grubs. However some recruitment function exist in the literature $[2,13,19,17]$. The widely used are: 
Beverton-Holt: $\quad f(x)=\alpha x /(1+\beta x)$

Ricker: $\quad f(x)=\alpha x e^{-\beta x}$

Power function: $\quad f(x)=\alpha x^{1-\beta}$

Shepherd: $\quad f(x)=\alpha x /\left(1+\beta x^{c}\right)(c>0)$

Saila-Lorda: $\quad f(x)=\alpha x^{\gamma} e^{-\beta x}$

Deriso-Schute: $\quad f(x)=\alpha x(1-\beta x)^{1 / \gamma}$

where $\alpha, \beta$ and $\gamma$ are positive real parameters.

We assume that the fish population is submitted to fishing action and that the harvested number fish during each period $[k, k+1)$ is available for measurement. The catch in the time interval period $[k, k+1)$ can be expressed as follows (see [7] page 146 for instance):

$$
y(k)=\sum_{i=1}^{n} \int_{k}^{k+1} q_{i} E(k) \tau x_{i}(t) d t
$$

After some computations, the relation (2) can be written:

$$
y(k)=\sum_{i=1}^{n} \frac{q_{i} E(k)}{q_{i} E(k)+M_{i}}\left(1-\exp \left(-\left(M_{i}+q_{i} E(k)\right) \tau\right)\right) x_{i}(k)
$$

In order to implement the dynamical system (1) we must know the initial condition $x\left(k_{0}\right)$ at a given time $k_{0}$, that is we have to know the number of fish in each class at time $k_{0}$. But it is a difficult task to count the number of a fish population in an ocean zone, all that one can measure is the value of the catch $y(k)$ for a given time $k$. The motivation of this paper is to assess the state $x_{i}(k)$ for a given fish population whose dynamics are described by the model (1) using the capture data(2). To solve this problem we use a classical method of control theory which consists in building an observer for the system (1). We briefly recall the meaning of an observer in control theory. Let us consider a discrete time system of the form :

$$
x(k+1)=G(x(k), u(k))
$$

with the measurable output:

$$
y(k)=O(x(k), u(k))
$$

Where $x(k) \in \mathbb{R}^{n}$ is the system state at time $k$ and $u(k)$ is a control term at time $k$. As we mentioned it above, in most models cases we do not always have access to the 
state $x(k)$ but we can measure only a function $y(k)$ of the state. This function is called the measurable output. An observer of the system (4) is an auxiliary dynamical system whose input is the input and the output of system (4), which produce an estimator $\hat{x}(k)$ of the state $x(k)$ such that the estimation error $x(k)-\hat{x}(k)$ tends towards zero as time $k$ goes to infinity and must remain small if it starts small. For example, for linear system of the form:

$$
\left\{\begin{aligned}
x(k+1) & =A x(k)+B u(k) \\
y(k) & =C x(k)
\end{aligned}\right.
$$

Where $x(k) \in \Omega \subset \mathbb{R}^{n}, u(k) \in \mathbb{R}^{m}, A \in \mathbb{R}^{n} \times \mathbb{R}^{n}, B \in \mathbb{R}^{n} \times \mathbb{R}^{m}, y(k) \in \mathbb{R}^{q}$ and $C \in$ $\mathbb{R}^{q} \times \mathbb{R}^{n}$, the synthesis of the observer is entirely determined by the Luenberger method [12] if the pair (A,C) is uniformly observable i.e if $\operatorname{rank}\left(\begin{array}{llll}C^{t} & A^{t} C^{t} & \ldots & A^{n-1} C^{t}\end{array}\right)=n$. In fact, the candidate observer is put as being

$$
\hat{x}(k+1)=A \hat{x}(k)+B u(k)+K(y(k)-C \hat{x}(k))
$$

It is shown that if the pair $(A, C)$ is observable one can choose $K$ such that the estimation error $x(k)-\hat{x}(k)$ tends towards zero when $k$ tends towards infinity. For discrete time nonlinear systems, the synthesis of an observer is a difficult task, only few results exist in the literature $([4],[5],[10],[14],[20],[23])$ and most of these results give local convergence of the observer. While it is difficult to build an observer for the model (1)(3) due to the high non linearity of the first equation one can use interval estimator theory to estimate the system states ([4],[8],[9]). In [8] an algorithm called "Interval Moving Horizon state Estimator" ( IMHSE) which builds an interval estimator state of a nonlinear dynamical system is developed. The algorithm IMHSE has the advantage of being applicable to a large class of dynamical system with global convergence. We would like to notice that an observer for system (1) with the measurable output (3) has been developed in [15]. The advantage of the observer of [15] is that it does not use the expression of the poorly known recruitment function but this observer uses the outputs at time $k+1$ instead of $k$ to estimate the system state at time $k+1$. However, an observer generally uses the past information outputs and inputs in order to give future estimation state of a given system. Since we are interested in the prediction of fish stock for better management of the resources, we must build an observer which uses only the past information to estimate future states of the model. Furthermore, contrary to the observer built in [15], the observer we construct in this paper requires no condition on the minimal fishing effort. Our paper is organized as follows: first Section is devoted to motivation problem and presentation of the fish model for which we aim to build an interval observer. In Section two we briefly recall the IMHSE algorithm and adapt the algorithm to the considered harvested fish model (1)(3). A numerical example is developed in the last Section to show the observer convergence. To simplify the notation let us denote equations (1) and (3) respectively by: 


$$
\begin{aligned}
& x(k+1)=F(x(k), E(k)) \\
& y(k)=H(x(k), E(k))
\end{aligned}
$$

Functions $F$ and $H$ are define by:

$$
\begin{aligned}
F: \mathbb{R}^{n} \times \mathbb{R} & \mapsto \mathbb{R}^{n} \\
(x(k), E(k)) & \mapsto\left(f\left(\sum_{i=1}^{n} b_{i} x_{i}(k)\right), v_{1}(k) x_{1}(k), \ldots, v_{n-1}(k) x_{n-1}(k)\right)^{t} \\
H: \mathbb{R}^{n} \times \mathbb{R} & \mapsto \mathbb{R} \\
(x(k), E(k)) & \mapsto \sum_{i=1}^{n} C_{i}(k) x_{i}(k)
\end{aligned}
$$

where

$$
\begin{aligned}
x(k) & =\left(x_{1}(k), x_{2}(k), \ldots, x_{n}(k)\right)^{t}, \\
v_{i}(k) & =\exp \left(-M_{i}-q_{i} E(k) \tau\right), i=1, \ldots, n-1, \\
C_{i}(k) & =\frac{q_{i} E(k)}{q_{i} E(k)+M_{i}}\left(1-\exp \left[-\left(M_{i}+q_{i} E(k)\right) \tau\right]\right), i=1, \ldots,
\end{aligned}
$$

\section{Observer synthesis: Interval Moving Horizon State Estimation (IMHSE)}

The algorithm IMHSE is well developed in [8], we briefly recall the algorithm description for system (8) with measurable output (9). Let $s h$ be the start horizon time and $l h$ the length horizon time observation. It is assumed that the measurable output $y(k)$ is available for $k=s h, \ldots, s h+l h-1$. The algorithm IMHSE uses the available output $y(k)$ to compute an interval estimator state of system (8). For a given $s h \in \mathbb{N}$ and $l h \in \mathbb{N}$, let $x(s h), x(s h+1), \ldots, x(s h+l h-1)$ the values of the unknown real states corresponding to the measurable outputs $y(s h), y(s h+1), \ldots, y(s h+l h-1)$. For any $z \in \Omega$, we define the quadratic function:

$$
j_{s h}(z)=\frac{1}{2} \sum_{k=s h}^{s h+l h-1}\left(H\left(z_{k}, E(k)\right)-y(k)\right)^{2}
$$

Where $z_{s h}=z$ and $z_{k}=F\left(z_{k-1}, E(k-1)\right)$ for $k=s h+1, \ldots, s h+l h-1$.

Let us consider the following inclusion functions :

$$
\begin{aligned}
\mathcal{F}: \mathcal{B}\left(\mathbb{R}^{n}\right) \times \mathbb{R} & \mapsto \mathcal{B}\left(\mathbb{R}^{n}\right) \\
(I, E) & \mapsto \mathcal{F}(I, E)=\{F(x, E) / x \in I\}
\end{aligned}
$$




$$
\begin{aligned}
\mathcal{J}_{\text {sh }}: \mathcal{B}\left(\mathbb{R}^{n}\right) & \mapsto \mathcal{B}(\mathbb{R}) \\
I & \mapsto \mathcal{J}_{\text {sh }}(I)=\left\{j_{\text {sh }}(x) / x \in I\right\}
\end{aligned}
$$

We wish to compute at time $s h+l h$ an interval with width less than $\epsilon$ (arbitrary chosen) that contains the solution $x(s h+l h)$ of system $(1)$ at time $s h+l h$. Consider the problem:

$$
\min _{z \in \Omega} j_{s h}(z)
$$

The first thing we have to do is to locate an invariant domain $\Omega$ of system (8) and to find in $\Omega$ all the intervals with width less than $\eta$ (arbitrary chosen) that contain a solution of problem (11) by the use of global optimization by interval analysis [11].

\subsection{Global optimization algorithm}

We describe a simple algorithm that globally minimizes the nonlinear function (10) [8]. The algorithm find all intervals with wide less than an arbitrary chosen positive real number.

let $\eta>0$ arbitrary chosen and $L$ a box list that contained solution of problem (11).

Step1 : Consider an invariant domain $\Omega$ for the model (1)

Initialize $L$ to be $\Omega$

Step2 : Compute $d$ the wide of $\Omega$

Step3 : $\left[L_{\Omega}, U_{\Omega}\right]:=\mathcal{J}_{s h}(\Omega)$ and put $U_{j}:=U_{\Omega}$

Step4 : While $d>\eta$ do:

Step4 - 1 : Remove the first box $X$ of the list $L$

Step4 : -2 Bisect $X$ by its widest such that $X=X_{1} \cup X_{2}$

Step4 $-3:\left[L_{X_{1}}, U_{X_{1}}\right]:=\mathcal{J}_{s h}\left(X_{1}\right)$

If $L_{X_{1}}>U_{j}$ then delete $X_{1}$

Else add $X_{1}$ at the end of the list $L$

If $X_{1}$ is listed,

$U J_{X_{1}}:=\mathcal{J}_{s h}\left(\beta_{X_{1}}\right)$, where $\beta_{X_{1}}$ is the midpoint of $X_{1}$ 
if $X_{1}$ is listed and $U J_{X_{1}}<U_{j}$ do $U_{j}:=U J_{X_{1}}$

Step4 $-4:\left[L_{X_{2}}, U_{X_{2}}\right]:=\mathcal{J}_{s h}\left(X_{2}\right)$

if $L_{X_{2}}>U_{j}$ then delete $X_{2}$

Else add $X_{2}$ at the end of the list $L$

If $X_{2}$ is listed

$U J_{X_{2}}:=\mathcal{J}_{s h}\left(\beta_{X_{2}}\right)$, where $\beta_{X_{2}}$ is the midpoint of $X_{2}$

If $X_{2}$ is listed and $U J_{X_{2}}<U_{j}$ do $U_{j}:=U J_{X_{2}}$

Step4 - 5: Allocate the widest of the next box $X$ in the list $L$ to d:

$$
d:=\operatorname{wide}(X)
$$

\subsection{IMHSE Algorithm}

The IMHSE algorithm computes an enclosure of the state estimation at time $s h+l h$ by using the global optimization algorithm. The algorithm is the following:

Step1 : Define the invariant domain $\Omega$ for the system (8).

Step2 : Solve the minimization problem (11) using the global optimization algorithm. Initialized $X_{s h}$ to the box with width less than the prescribe tolerance $\eta$ that contained solution of problem (11).

Step3 : Compute the interval estimator vector $X_{s h+l h}$ at the end of the time horizon:

$$
X_{s h+l h}=\underbrace{\mathcal{F} O \mathcal{F} \ldots \mathcal{F}}_{l h-1}\left(X_{s h}\right)
$$

Step4 : Go to step 2 and calculate the next interval estimator: $s h:=s h+1$

See $[8]$ for more details on this algorithm.

Definition $1\left(X_{k}\right)_{k \in \mathbb{N}} \subset \mathcal{B}\left(\mathbb{R}^{n}\right)$ is an interval observer of system (1) if there exists $k_{0} \in \mathbb{N}$ such that $\forall k \geq k_{0} x(k) \in X_{k}$, where $x(k) k \geq k_{0}$ is the solution of (1) for a given solution initial state $x\left(k_{0}\right)$.

Consider the following hypothesis:

$\mathcal{H}_{1}$ : the model $(F, H)$ in $(8),(9)$ is perfect. 
$\mathcal{H}_{2}:$ the model $(F, H)$ is free noise.

$\mathcal{H}_{1}$ and $\mathcal{H}_{2}$ have no biological sens, we give these hypotheses in order to have a well posed mathematical problem.

Proposition 1 The sequence box $\left(X_{k}\right)_{k \in \mathbb{N}}$ generated by the IMHSE algorithm is an interval observer of (8).

As we have mentioned it above, the result in Proposition 1 is given in [8], we adapt the proof to the harvested fish model (1),(3). To this end, let us consider the following lemma:

Lemma 1 Assume that $\mathcal{H}_{1}, \mathcal{H}_{2}$ are satisfied and that the system (8) with measurable output (9) is locally observable then the problem (11) has a unique optimal solution which corresponds to the solution of (8) at the beginning of the time horizon sh.

The proof of this lemma is given in [3].

Proof: (Proposition 1)

We restrict the proof to the case where $n=3$ and the fishing effort $E(k)=E$ is constant. Where $n>3$ and considering a time varying fishing effort the proof is similar but the computation are too long.

Let $x(s h)$ the solution at time $s h$ of system (8).

$\mathcal{H}_{1}$ and $\mathcal{H}_{2}$ imply that $j_{s h}(x(s h))=0$ and thus $x(s h)$ is a global minimum of the positive function $j_{s h}$.

Now let $x(k)$ and $\bar{x}(k), k \geq 0$ be two solutions of (1) such that:

$$
H(x(k), E)=H(\bar{x}(k), E), \forall k \geq 0
$$

We note $z(k)=x(k)-\bar{x}(k)$ and we obtain:

$$
C_{1} z_{1}(k)+C_{2} z_{2}(k)+C_{3} z_{3}(k)=0 .
$$

$$
C_{1} z_{1}(k+1)+C_{2} z_{2}(k+1)+C_{3} z_{3}(k+1)=0
$$

$$
C_{1} z_{1}(k+2)+C_{2} z_{2}(k+2)+C_{3} z_{3}(k+2)=0
$$

Let $\alpha_{1}=\frac{C_{2} v_{1}}{C_{1}}$ and $\alpha_{2}=\frac{C_{3} v_{2} v_{1}}{C_{1}}$, then (14) imply that

$$
z_{1}(k+2)+\alpha_{1} z_{1}(k+1)+\alpha_{2} z_{1}(k)=0
$$


Hence for $k=0$, we can write:

$z_{1}(2)=-\alpha_{1} z_{1}(1)-\alpha_{2} z_{1}(0)$

For $k=1$, we obtain:

$$
\begin{aligned}
& z_{1}(3)=-\alpha_{1} z_{1}(2)-\alpha_{2} z_{1}(1)=-\alpha_{1}\left(-\alpha_{1} z_{1}(1)-\alpha_{2} z_{1}(0)\right)-\alpha_{2} z_{1}(1) \\
& \left.z_{1}(3)=\left(\alpha_{1}^{2}-\alpha_{2}\right) z_{1}(1)+\alpha_{1} \alpha_{2} z_{1}(0)\right) \\
& z_{1}(3)=f_{0}(x(2))-f_{0}(\bar{x}(2)) \quad \text { where } f_{0}(x)=f\left(\sum_{i=1}^{n} b_{i} x_{i}\right) \\
& z_{1}(3)=f_{0}\left(x_{1}(2), v_{1} x_{1}(1), v_{1} v_{2} x_{1}(0)\right)-f_{0}\left(\bar{x}_{1}(2), v_{1} \bar{x}_{1}(1), v_{1} v_{2} \bar{x}_{1}(0)\right) \\
& z_{1}(3)=\left(x_{1}(2)-\bar{x}_{1}(2)\right) G_{1}+v_{1}\left(x_{1}(2)-\bar{x}_{1}(2)\right) G_{2}+v_{1} v_{2}\left(x_{1}(0)-\bar{x}_{1}(0)\right) G_{3}
\end{aligned}
$$

where

$$
G_{i}=\int_{0}^{1} \frac{\partial f_{0}}{\partial x_{i}}\left(t z_{1}(2)+\bar{x}_{1}(2), t v_{1} z_{1}(1)+v_{1} \bar{x}_{1}(1), t v_{1} v_{2} z_{1}(0)+v_{1} v_{2} \bar{x}_{1}(0)\right) d t,
$$

for $i=1,2,3$

Then we have:

$$
\begin{aligned}
& z_{1}(3)=z_{1}(2) G_{1}+v_{1} z_{1}(1) G_{2}+v_{1} v_{2} z_{1}(0) G_{3} \\
& z_{1}(3)=G_{1}\left(-\alpha_{1} z_{1}(1)-\alpha_{2} z_{1}(0)\right)+v_{1} z_{1}(1) G_{2}+v_{1} v_{2} z_{1}(0) G_{3} \\
& z_{1}(3)=\left(-\alpha_{1} G_{1}+v_{1} G_{2}\right) z_{1}(1)+\left(-\alpha_{2} G_{1}+v_{1} v_{2} G_{3}\right) z_{1}(0)
\end{aligned}
$$

Remark that $G_{i}=\frac{b_{i}}{b_{1}} G_{1}$, we finally obtain:

$$
\left(v_{1} v_{2} G_{3}-\alpha_{2} G_{1}-\alpha_{1} \alpha_{2}\right) z_{1}(0)+\left(v_{1} G_{2}-\alpha_{1} G_{1}-\alpha_{1}^{2}+\alpha_{2}\right) z_{1}(1)=0
$$

If $k=2$, equation (15) gives:

$$
\begin{aligned}
& z_{1}(4)=-\alpha_{1} z_{1}(3)-\alpha_{2} z_{1}(2)=-\alpha_{1}\left(\left(\alpha_{1}^{2}-\alpha_{2}\right) z_{1}(1)+\alpha_{1} \alpha_{2} z_{1}(0)\right)-\alpha_{2}\left(-\alpha_{1} z_{1}(1)-\alpha_{2} z_{1}(0)\right) \\
& z_{1}(4)=\alpha_{2}\left(\alpha_{2}-\alpha_{1}^{2}\right) z_{1}(0)+\alpha_{1}\left(2 \alpha_{2}-\alpha_{1}^{2}\right) z_{1}(1)
\end{aligned}
$$

Otherwise

$z_{1}(4)=f_{0}(x(3))-f_{0}(\bar{x}(3))$ 


$$
\begin{aligned}
& z_{1}(4)=f_{0}\left(x_{1}(3), v_{1} x_{1}(2), v_{1} v_{2} x_{1}(1)\right)-f_{0}\left(\bar{x}_{1}(3), v_{1} \bar{x}_{1}(2), v_{1} v_{2} \bar{x}_{1}(1)\right) \\
& z_{1}(4)=\left(x_{1}(3)-\bar{x}_{1}(3)\right) \bar{G}_{1}+v_{1}\left(x_{1}(2)-\bar{x}_{1}(2)\right) \bar{G}_{2}+v_{1} v_{2}\left(x_{1}(1)-\bar{x}_{1}(1)\right) \bar{G}_{3} \\
& z_{1}(4)=z_{1}(3) \bar{G}_{1}+v_{1} z_{1}(2) \bar{G}_{2}+v_{1} v_{2} z_{1}(1) \bar{G}_{3} \\
& \text { where }
\end{aligned}
$$$$
\bar{G}_{i}=\int_{0}^{1} \frac{\partial f_{0}}{\partial x_{i}}\left(t z_{1}(3)+\bar{x}_{1}(3), t v_{1} z_{1}(2)+v_{1} \bar{x}_{1}(2), t v_{1} v_{2} z_{1}(1)+v_{1} v_{2} \bar{x}_{1}(1)\right) d t, \quad i=1,2,3
$$

Since $\left.z_{1}(3)=\left(\alpha_{1}^{2}-\alpha_{2}\right) z_{1}(1)+\alpha_{1} \alpha_{2} z_{1}(0)\right)$ and $z_{1}(2)=-\alpha_{1} z_{1}(1)-\alpha_{2} z_{1}(0)$, we obtain: $z_{1}(4)=\left(\left(\alpha_{1}^{2}-\alpha_{2}\right) z_{1}(1)+\alpha_{1} \alpha_{2} z_{1}(0)\right) \bar{G}_{1}+\left(-\alpha_{1} z_{1}(1)-\alpha_{2} z_{1}(0)\right) v_{1} \bar{G}_{2}+v_{1} v_{2} z_{1}(1) \bar{G}_{3}$ $z_{1}(4)=\left(\left(\alpha_{1}^{2}-\alpha_{2}\right) \bar{G}_{1}-\alpha_{1} v_{1} \bar{G}_{2}+v_{1} v_{2} \bar{G}_{3}\right) z_{1}(1)+\left(\alpha_{1} \alpha_{2} \bar{G}_{1}-\alpha_{2} v_{1} \bar{G}_{2}\right) z_{1}(0)$ The two expressions of $z_{1}(k)$ give:

$$
\begin{aligned}
& \left(\alpha_{1} \alpha_{2} \bar{G}_{1}-\alpha_{2} v_{1} \bar{G}_{2}-\alpha_{2}\left(\alpha_{2}-\alpha_{1}^{2}\right)\right) z_{1}(0) \\
& +\left(\alpha_{1}\left(\alpha_{1}^{2}-2 \alpha_{2}\right)+\left(\alpha_{1}^{2}-\alpha_{2}\right) \bar{G}_{1}-\alpha_{1} v_{1} \bar{G}_{2}+v_{1} v_{2} \bar{G}_{3}\right) z_{1}(1)=0
\end{aligned}
$$

Remark that $\bar{G}_{i}=\frac{b_{i}}{b_{1}} \bar{G}_{1}$, for $i=2,3$, hence equation (17) can be written as:

$$
\begin{aligned}
& \left(\left(\alpha_{1} \alpha_{2}-\alpha_{2} v_{1} \frac{b_{2}}{b_{1}}\right) \bar{G}_{1}-\alpha_{2}\left(\alpha_{2}-\alpha_{1}^{2}\right)\right) z_{1}(0) \\
& +\left(\alpha_{1}\left(\alpha_{1}^{2}-2 \alpha_{2}\right)+\left(\left(\alpha_{1}^{2}-\alpha_{2}\right)-\alpha_{1} v_{1} \frac{b_{2}}{b_{1}}+v_{1} v_{2} \frac{b_{3}}{b_{1}}\right) \bar{G}_{1}\right) z_{1}(1)=0
\end{aligned}
$$

Coupling equation (16) and (18) we obtain the following system:

$$
\left\{\begin{array}{l}
\left(\left(v_{1} v_{2} \frac{b_{3}}{b_{1}}-\alpha_{2}\right) G_{1}-\alpha_{1} \alpha_{2}\right) z_{1}(0)+\left(\left(v_{1} \frac{b_{2}}{b_{1}}-\alpha_{1}\right) G_{1}-\alpha_{1}^{2}+\alpha_{2}\right) z_{1}(1)=0 \\
\left(\left(\alpha_{1} \alpha_{2}-\alpha_{2} v_{1} \frac{b_{2}}{b_{1}}\right) \bar{G}_{1}-\alpha_{2}\left(\alpha_{2}-\alpha_{1}^{2}\right)\right) z_{1}(0) \\
\quad+\left(\alpha_{1}\left(\alpha_{1}^{2}-2 \alpha_{2}\right)+\left(\left(\alpha_{1}^{2}-\alpha_{2}\right)-\alpha_{1} v_{1} \frac{b_{2}}{b_{1}}+v_{1} v_{2} \frac{b_{3}}{b_{1}}\right) \bar{G}_{1}\right) z_{1}(1)=0
\end{array}\right.
$$


Let:

$$
\begin{aligned}
\Gamma_{11}= & v_{1}(0) v_{2}(1) \frac{b_{3}}{b_{1}} G_{1}-\alpha_{2}(0) G_{1}-\alpha_{1}(1) \alpha_{2}(0) \\
\Gamma_{12}= & v_{1}(1) \frac{b_{2}}{b_{1}} G_{1}-\alpha_{1}(0) G_{1}-\alpha_{1}(0) \alpha(1) \\
\Gamma_{21}= & \alpha_{1}(1) \alpha_{2}(0) \bar{G}_{1}-\alpha_{2}(0) v_{1}(2) \frac{b_{2}}{b_{1}} \bar{G}_{1}-\alpha_{2}(0)\left(\alpha_{2}(2)-\alpha_{1}(2) \alpha_{1}(1)\right) \\
\Gamma_{22}= & \left(\alpha_{1}(0) \alpha_{1}(1)-\alpha_{2}(1)\right) \bar{G}_{1}-\alpha_{1}(0) v_{1}(2) \frac{b_{2}}{b_{1}} \bar{G}_{1}+v_{1}(1) v_{2}(2) \frac{b_{3}}{b_{1}} \bar{G}_{1} \\
& -\left(\alpha_{2}(2) \alpha_{1}(0)-\alpha_{1}(2)\left(\alpha_{1}(0) \alpha_{1}(1)-\alpha_{2}(1)\right)\right)
\end{aligned}
$$

We write system (19) in the condensed form:

$$
\left\{\begin{array}{l}
\Gamma_{11} z_{1}(0)+\Gamma_{12} z_{1}(1)=0 \\
\Gamma_{21} z_{1}(0)+\Gamma_{22} z_{1}(1)=0
\end{array}\right.
$$

Assume that the parameters of the model are such that:

$$
-\Gamma_{21} \Gamma_{12}+\Gamma_{11} \Gamma_{22} \neq 0
$$

Then we obtain $z_{1}(0)=z_{1}(1)=0$. Using equation (15), we obtain $z_{1}(k)=0 \forall k \geq 0$. Remark that $z_{2}(2)=v_{1} z_{1}(1)$, hence $z_{2}(2)=0$.

By equation (12) we obtain $z_{3}(2)=0$ and consequently $z_{2}(1)=0$ and $z_{3}(1)=0$. $z_{3}(1)=0$ implies $z_{2}(0)=0$. Hence by equation (12), we have $z_{3}(0)=0$.

Finally $z_{1}(0)=z_{2}(0)=z_{3}(0)=0$. Hence if (21) holds then the model $(1)(3)$ is observable. By lemma 1, the solution of problem (11) is unique. Hence the Global Optimization Algorithm computes a unique interval $X_{s h}$ that contains $x(s h)$.

$F^{l h-1}$ is a continuous function. Hence,

$\exists \gamma>0 /\left\|x-x_{s} h\right\|<\gamma \Rightarrow\left\|F^{l h-1}(x, E)-F^{l h-1}\left(x_{s} h, E\right)\right\|<\frac{\eta}{2}$

The interval $X_{s h}$ is such that its diameter is less than $\eta$. By the inclusion function $\mathcal{F}$, we obtain:

$$
X_{l h}=\mathcal{F}^{l h-1}\left(X_{s h}\right) \text { and wide }\left(X_{l h}\right)<\eta
$$

\subsection{Numerical example}

We consider the Beverton-Holt recruitment function:

$$
f(x)=\alpha x /(1+\beta x)
$$

Assume that the length of the time observation $l h$ is equal to 3 i.e the past measurable output $y(k-3), y(k-2), y(k-1)$ are needed to calculate the interval estimator $X_{k}$ 
of the state $x(k)$ at a given time $k$. Let us consider the following parameter values (these values are not from real data):

$$
\begin{array}{ll}
\alpha=1, \beta=0.0002, & b=\left[\begin{array}{lll}
5 & 5 & 5
\end{array}\right], \\
q=\left[\begin{array}{lll}
0.24 & 0.36 & 0.42
\end{array}\right], & M=\left[\begin{array}{lll}
0.2 & 0.2 & 0.2
\end{array}\right], \\
\tau=1, & E(k)=E=8 / 3,
\end{array}
$$

We verify that with these parameters, the relation (21) becomes

$$
0.00734219+0.0055823 G_{1}+0.00140772 \bar{G}_{1}+0.0025079 G_{1} \bar{G}_{1} \neq 0
$$

Relation (22) is true because $G_{1}$ and $\bar{G}_{1}$ are positive reals when $f$ is the Beverthon-Holt recruitment function. The system is then observable and the IMHSE algorithm computes an interval observer for the model. With the Beverthon-Holt recruitment function $f$ one can remark that $\frac{1}{\beta}$ is a global maximum of the map: $x \mapsto f(x)$ on $\mathbb{R}$.

Let:

$$
m=\min _{i} M_{i} \text { and } q=\min _{i} q_{i}
$$

For all $k>0$, we have:

$$
\begin{aligned}
& x_{1}(k)=f\left(\sum_{i=1}^{3} b_{i} x(k-1)\right) \leq \frac{1}{\beta} \\
& x_{2}(k)=e^{-M_{1}-q_{1} E \tau} x_{1}(k-1) \leq e^{-m-q E \tau} \cdot \frac{1}{\beta} \\
& x_{3}(k)=e^{-M_{2}-q_{2} E \tau} x_{2}(k-1) \leq e^{-2 m-2 q E \tau} \cdot \frac{1}{\beta}
\end{aligned}
$$

It becomes easy now to see that: $\Omega=\left[0, \frac{1}{\beta}\right] \times\left[0, \frac{e^{-m-q E \tau}}{\beta}\right] \times\left[0, \frac{e^{-2 m-2 q E \tau}}{\beta}\right]$ is an invariant domain for (1) when $n=3$. Simulations are done with Scilab. The real states are represented by solid line and the bound observer are represented by the dashed one. $\mathrm{Re}$ sults of simulations are drown in Figure 1 and the convergence of the interval observer is very fast. Various modifications of the parameters given in simulation will note influence the observer convergence while the relation (21) is satisfy. 

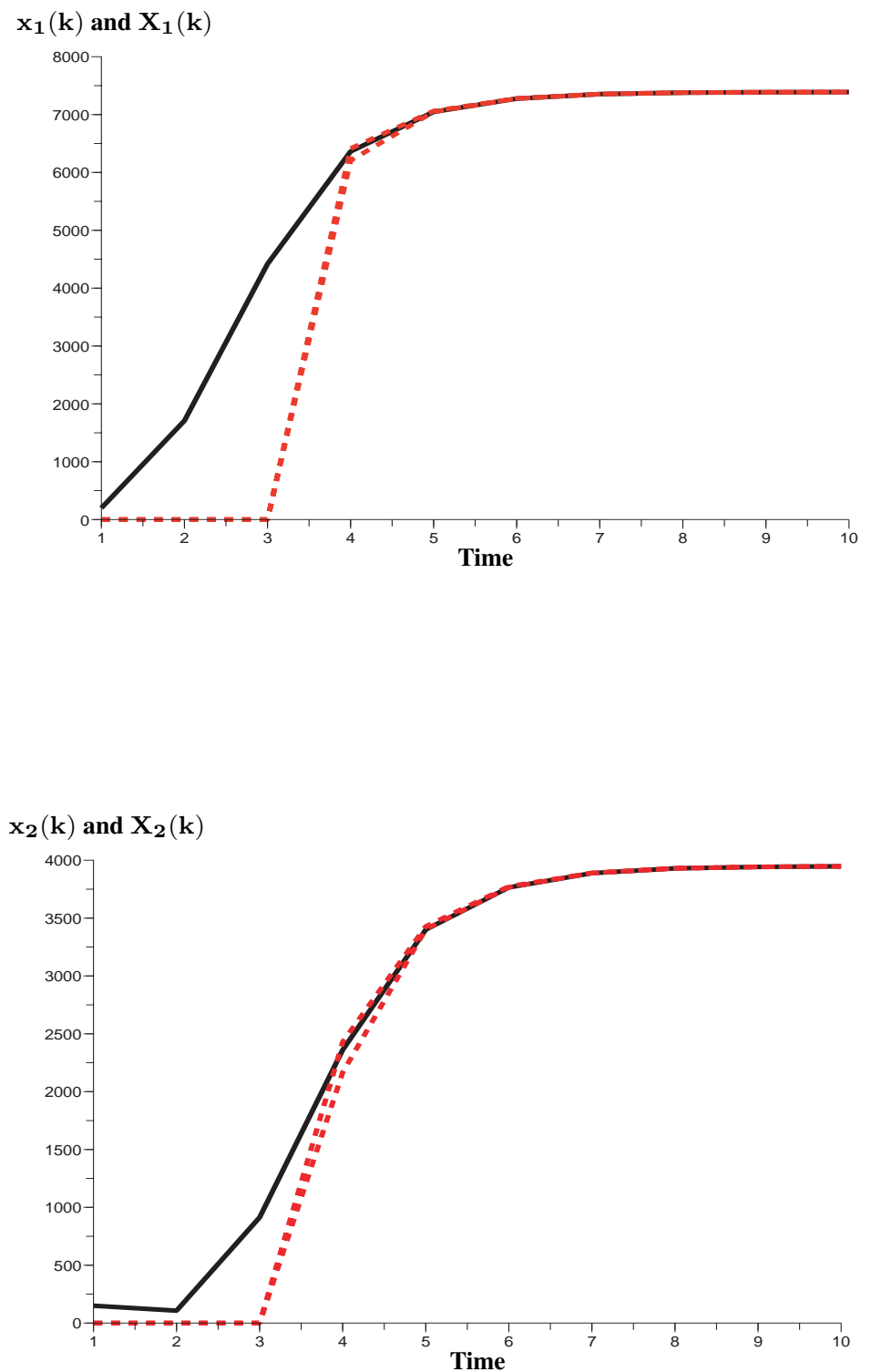


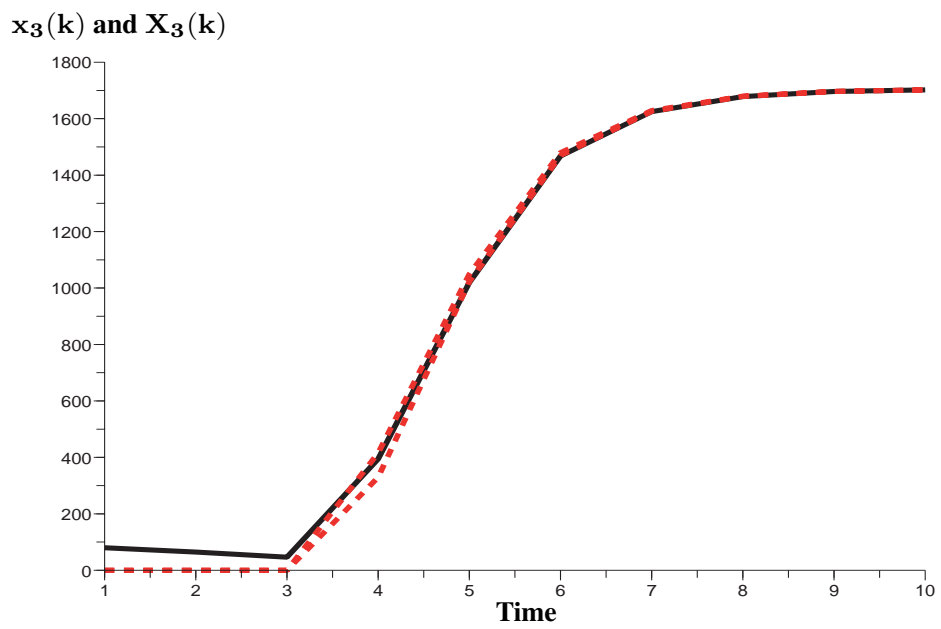

Figure 1: state $x$ (solid line) and its interval observer X (dashed line)

\section{Conclusion}

We have applied the IMHSE algorithm by considering a discrete time nonlinear model for a harvested fish population. We note that the algorithm works for any $n$-age class and any recruitment function when it is possible to find an invariant domain for the model (1). The IMHSE algorithm works well due to the observability of the model (1)(3). Some numerical examples are given and one can see on simulation that the convergence of the interval observer is very fast. The importance of this algorithm application is that it can help to predict the stock evolution for a given fish population whose dynamics are described by the model (1-3) using only the measurable output. Hence the numerical interval estimator can be helpful in management policies. This work is an example that shows how control theory combined with computer science can be used as a tool for the management of renewable resources.

\section{References}

[1] A.El Assoudi, E. H. El YaAgoubi, AND H. Hammouri., "Non-linear observer based on the Euler discretization", Internat. J. Control, 75(14):784-791, 2002.

[2] R. J. H. Beverton, And S. J. Holt, On The Dynamics of Exploited Fish Populations, Chapman \& Hall, London, 1957.

[3] L. BoillereauX, J. M. Flaus., “A New Approach for Designing Model-Based indirect Sensors”, IEEE Transactions on Control systems Technology, Vol. 8, No. 4, JULY 2000. 
[4] G. Chen, J. WANG, L.S. ShIEH., "Interval Kalman Filtering", IEEE Transactions on Aerospace and electronic Systems, 33 (1),250-259, 1997.

[5] G. Ciccarella, M. Dalla Mora and A. Germani., "Observers for discrete-time nonlinear systems", Systems Control Lett, 20 5, 373-382, 1993.

[6] W. Doubleday, Harvesting in matrix population models, Biometrics, 31, pp:189-200, 1975.

[7] W. M. Getz and R. G. Haight., "Population harvesting. Demographic models of fish, forest, and animal resources.", volume 27 of Monographs in Population Biology. Princeton University Press, Princeton, NJ, 1989.

[8] H. V. GonzÁles, J. M. Flaus, G. ACuna., "Moving horizon state estimation with global convergence using interval techniques: application to biotechnological processes", Journal of Process Process Control, 14, 325-336, 2003

[9] L. JAULIN, E. WALTER, "Guaranteed bounded-error parameter estimation for non-linear models with uncertain experimental factors", Automatica, 35, 849-856, 1999.

[10] I. KARAFYLLIS AND C. KRAVARIS, "On the observer problem for discrete-time control systems", IEEE Transactions on Automatic Control, 52(1):14-25, 2007.

[11] A. P. LECLERC, Efficient and Reliable Global Optimization, PhD Thesis. The Ohio State University. 1992.

[12] D. Luenberger, “An introduction to observers”, IEEE Transaction on Automatic Control, AC-16 (6), 596-602, 1971.

[13] P. Magal and D. Pelletier, "A fixed point theorem with application to a model of population dynamics“, J. Difference Equ. Appl., 3, pp:65-87, , 1997.

[14] P. MoraAl And J. GrizzLe, "Observer design for nonlinear systems with discrete-time measurements", IEEE Trans. Autom. Control, 40(3):395-404, 1995.

[15] D. NGon, A. Iggidr, A. Guiro And A. OuAhbi, "An Observer for a nonlinear agestructured model of a harvested fish population", Mathematical Biosciences and Engineering, 5(2), 337-354, 2008.

[16] D. Pelletier And P. Magal, "Dynamics of a migratory population under different fishing effort allocation schemes in time and space", Canadian Journal of Fisheries and Aquatic sciences, 53, pp:1189-1199, 1996.

[17] W. E. RICKER, Stock and recruitment, J. Fish. Res. Board Can., 11, pp:559-623, 1954.

[18] S. SAILA AND E. LORDA, "Sensitivity analysis applied to a matrix Model of the Hudson river Striped bass population", In W. van Winkle, editor. Proceedings of conference on assessing the effects of power-plant induced mortality on fish population., Pergamon Press, 20, pp:311-332, 1977.

[19] J. G. SHEPHERD, “A family of general production curves for exploited populations", Mathematical Biosciences, 59, pp:77-93, 1982.

[20] Y. SONG AND J.W. GRIZZLE, "The extended Kalman filter as a local asymptotic observer for discrete time nonlinear systems", J. of Mathematical Systems,Estimation, and Control, No.1, 59-78, 1995.

[21] S. Touzeau, Modèles de contrôle en gestion des pêches. PhD thesis, Thèse de Doctorat de l’Université de Nice-Sophia Antipolis, Science de l’ingénieur (France), 266 p, 1997. 
[22] S. Tuljapurkar AND H. CASWell, Structured population models in marine, terrestrial, and fresh-water systems: 1993 Summer school at Cornell university. Population and community biology series 18, Chapman Hall, Summer school on Structured population models, Ithaca (NY US),643 pp, 1997.

[23] M. Xiao, N. Kazantzis, C. Kravaris, And A. J. Krener, "Nonlinear discrete-time observer design with linearizable error dynamics", IEEE Transactions on Automatic Control, 48(4):622-626, 2003. 